\title{
Controle Adaptativo de Potência e Seleção de Usuários Para Maximização de Vazão em Sistema NOMA MIMO Multiusuário
}

\author{
Marcos N. L. de Carvalho ${ }^{1}$, Álisson A. Cardoso ${ }^{1}$, Flávio H. T. Vieira ${ }^{1}$ \\ ${ }^{1}$ Grupo Incomm, Escola de Engenharia Elétrica, Mecânica e de \\ Computação, Universidade Federal de Goiás (UFG), GO
}

\{marcosnlc4, alsnac\}@gmail.com, flavio_vieira@ufg.br

\begin{abstract}
The combination of Non-Orthogonal Multiple Access (NOMA) and Multiple Input and Multiple Output (MIMO) systems is considered a promising approach for 5G mobile communication networks. However, some simulation results indicate that optimization techniques, in this case, user selection, could be applied to improve some parameters of system performance. In this context, we propose an adaptive power allocation algorithm in conjunction with user selection for the downlink of a NOMA MIMO system in order to maximize the users' throughput. In order to evaluate the performance of the proposed algorithm, simulations were performed considering different power allocation algorithms of the users.

Resumo: A junção de técnicas de Acesso Múltiplo Não-Ortogonal (NOMA - Non-Orthogonal Multiple Access) e de sistemas com Múltiplas Entradas e Múltiplas Saídas (MIMO - Multiple Input and Multiple Output) é considerada uma abordagem promissora para as redes de comunicação móveis 5G. Entretanto, alguns resultados de simulações apontam que técnicas de otimização, no caso, seleção de usuários, poderiam ser aplicadas para melhorar alguns parâmetros de desempenho do sistema. Nesse contexto, propõe-se neste trabalho um algoritmo de alocação adaptativa de potência em conjunto com seleção de usuários para o enlace de descida de um sistema NOMA MIMO com o objetivo de maximizar a vazão de dados para os usuários. Para avaliar o desempenho do algoritmo proposto, foram realizadas simulações considerando diferentes algoritmos de alocação de potência dos usuários.
\end{abstract}

Keywords: 5G Networks; NOMA; MIMO; Power Control; User Selection.

Palavras-chaves: Redes 5G; NOMA; MIMO; Controle de Potência; Seleção de Usuários.

\section{INTRODUÇÃO}

O desenvolvimento de novas tecnologias de comunicação móvel foi motivado por requisitos cada vez maiores referentes à taxa de transmissão, confiabilidade e latência. Além da melhoria de outras características, o 5G também tem como desafio a otimização da vazão de dados dos usuários (Luo and Zhang, 2016).

Alguns pesquisadores acreditam que o esquema de modulação do 4G LTE, Modulação por Divisão de Frequência Ortogonal (OFDM - do inglês, Orthogonal FrequencyDivision Multiplexing), o qual utiliza o Acesso Múltiplo Ortogonal (OMA - do inglês, Orthogonal Multiple Access), talvez não seja o mais adequado para atender às necessidades do $5 \mathrm{G}$ por causa de limitações, como confinamento espectral ruim devido à elevada emissão fora de banda, problemas com comunicações de alta mobilidade, dentre outras (Luo and Zhang, 2016). Ademais, o OFDM faz uso do prefixo cíclico para garantir a ortogonalidade entre as portadoras, o que dificulta a redução da latência (Luo and Zhang, 2016). Portanto, há a necessidade de se encontrar técnicas de modulação e multiplexação que sejam mais adequadas para a realidade do $5 \mathrm{G}$.
Dentre os esquemas de modulação existentes, o Acesso Múltiplo Não Ortogonal (NOMA - do inglês, NonOrthogonal Multiple Access) vem se destacando como um forte candidato para ser utilizado nas redes $5 \mathrm{G}$ devido às suas vantagens, como a compatibilidade com sistemas de Múltiplas Entradas e Múltiplas Saídas (MIMO - do inglês, Multiple Input Multiple Output), a qual possibilita que múltiplos usuários sejam servidos simultaneamente, melhorando o desempenho da rede. Ao contrário do OFDM, o NOMA não exige a ortogonalidade de subportadoras ou dos sinais para realizar a multiplexação dos sinais dos usuários. A ideia chave do NOMA é explorar o domínio da potência para realizar a multiplexação dos sinais, empregando a técnica de Cancelamento Sucessivo de Interferência (SIC - do inglês, Successive Interference Cancelling) nos receptores dos usuários (Luo and Zhang, 2016) (Ali et al., 2016b) (Ding et al., 2016) (Islam et al., 2017).

Nesse contexto, propõe-se neste artigo um esquema de alocação adaptativa de potência em conjunto com um algoritmo de seleção de melhores usuários para o enlace de descida de um sistema NOMA MIMO com o objetivo de maximizar a vazão dos usuários. Como aborda-se um sistema multiusuário, considera-se a utilização do método de Diagonalização de Blocos com Zero-Forcing descrito 
em (Cho et al., 2010) para o cancelamento de interferências entre usuários. O desempenho do algoritmo proposto de alocação adaptativa de potência para os usuários foi comparado com outros esquemas como: alocação fixa, aleatória, algoritmo de (Ali et al., 2016a) e o algoritmo descrito em (Zamani et al., 2018). Para tanto, implementouse um sistema NOMA MIMO em MATLAB ${ }^{\circledR}$ capaz de simular diferentes configurações do sistema com múltiplos usuários.

\section{TRABALHOS RELACIONADOS}

Recentemente, a aplicação de NOMA em sistemas MIMO multiusuário tem atraído uma atenção significativa dos pesquisadores. Em (Zeng et al., 2017), compara-se o desempenho do NOMA com o do OMA em um cenário MIMO com dois usuários utilizando clusterização e beamforming (formação de feixe) provando, até mesmo analiticamente, que o MIMO-NOMA é mais eficiente em termos de taxa de transmissão agregada.

Em (Saito et al., 2013), analisa-se o desempenho de NOMA MIMO em uma transmissão de enlace de descida com alocação de potência fixa para os usuários com dois Equipamentos de Usuário de antena única e Estação Base com duas antenas de transmissão.

Outro importante estudo envolvendo alocação de potência em sistema NOMA MIMO é apresentado em (Ali et al., 2016a), no qual realizou-se uma simulação com um sistema NOMA MIMO, onde o número de antenas de transmissão, antenas de recepção na célula e EUs foram 3, 9 e 9, respectivamente. Em (Zamani et al., 2018), assim como no presente trabalho, os autores também propõem alocação dinâmica de potência com o objetivo de maximizar a vazão de dados dos usuários.

\section{CARACTERÍSTICAS DO NOMA}

O NOMA introduz a multiplexação de usuário por divisão de potência, explorando a diferença de ganho de canal entre usuários. Dessa forma, vários usuários são emparelhados, compartilhando os mesmos recursos, seja em tempo, frequência ou em código.

Para possibilitar a multiplexação por potência, o NOMA faz uso da técnica de Cancelamento Sucessivo de Interferência (SIC - do inglês, Successive Interference Cancellation). Nessa técnica, todos os sinais são sobrepostos no transmissor e, ao chegarem no receptor, o algoritmo de SIC decodifica o sinal de maior potência, removendo-se o primeiro sinal decodificado do sinal recebido. Caso a decodificação seja perfeita, a forma de onda com os demais sinais são obtidos precisamente. Repete-se o processo até se encontrar o sinal desejado (Kizilirmak, 2016). Uma ilustração do SIC é apresentada na Figura 1.

No enlace de descida (aplica-se uma ideia similar na abordagem para o enlace de subida), a Estação Base (EB) seleciona diferentes usuários sob os mesmos recursos, mas seus respectivos sinais são transmitidos utilizando diferentes níveis de potência. Explorando essas diferenças de potência, cada Equipamento de Usuário (EU) pode aplicar SIC e, por sua vez, decodificar o sinal desejado, tratando os demais sinais como interferências (Kizilirmak, 2016) (Ali et al., 2016b).

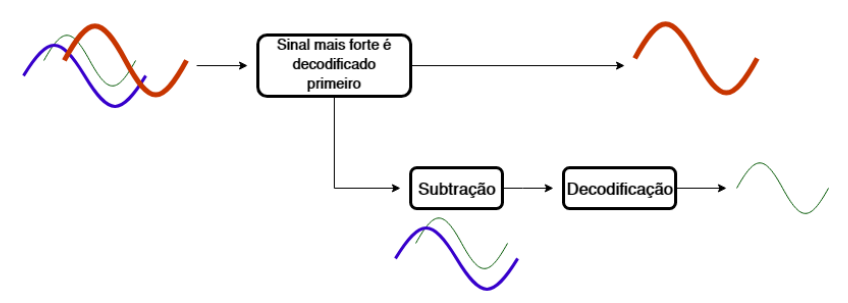

Figura 1. Ilustração da Técnica SIC. Adaptado de (Kizilirmak, 2016).

O sinal sobreposto transmitido pela EB e o sinal recebido pelo k-ésimo EU são representados pelas Equações (1) e (2), respectivamente (Kizilirmak, 2016).

$$
\begin{aligned}
x(t) & =\sum_{k=1}^{K} \sqrt{P_{k}} x_{k}(t) \\
y_{k}(t) & =x(t) g_{k}+w_{k}(t)
\end{aligned}
$$

onde $x_{k}$ é o sinal destinado ao k-ésimo EU, $y_{k}$ é o sinal recebido pelo $k$-ésimo EU, $g_{k}$ é o fator de atenuação do canal entre o k-ésimo EU e a EB, $w_{k}$ é o ruído gaussiano aditivo branco no $k$-ésimo EU com média zero e densidade espectral de potência $N_{0}(\mathrm{~W} / \mathrm{Hz})$. A potência do sinal do $k$-ésimo EU é dada por $P_{k}=\alpha_{k} P_{T}$, onde $\alpha_{k}$ é o coeficiente de alocação de potência do $k$-ésimo $\mathrm{EU}, P_{T}$ é a potência total do sinal transmitido (Ali et al., 2016b) (Luo and Zhang, 2016).

\section{MODELO DE TRANSMISSÃO EM SISTEMAS MIMO MULTIUSUÁRIO}

A principal dificuldade na transmissão em canais broadcast consiste no fato de a detecção coordenada dos sinais no receptor não ser direta, exigindo cancelamento de interferência na EB (Cho et al., 2010). São descritos em (Cho et al., 2010) vários métodos de transmissão para sistemas MIMO multiusuário. Neste trabalho, abordaremos somente a técnica de Diagonalização de Blocos com ZeroForcing para o enlace de descida de um modelo NOMA MIMO multiusuário (Cho et al., 2010).

Consideremos $K$ EUs independentes em um sistema MIMO. A EB e a cada EU são equipadas com $N_{B}$ e $N_{U}$ antenas, respectivamente. Sejam $\boldsymbol{x} \in \mathbb{C}^{N_{B} \times 1}$ o sinal transmitido pela $\mathrm{EB}$ e $\boldsymbol{y}_{u} \in \mathbb{C}^{N_{U} \times 1}$ o sinal recebido pelo $u$-ésimo $\mathrm{EU}, u=1,2, \ldots, K$. O sinal recebido pelo $u$-ésimo EU é dado por: $\boldsymbol{y}_{u}=\boldsymbol{H}_{u}^{D L} \boldsymbol{x}+\boldsymbol{z}_{u}, u=1,2, \ldots, K$, onde $\boldsymbol{H}_{u}^{D L} \in \mathbb{C}^{N_{M, u} \times N_{B}}$ é a matriz do canal entre a EB e o $u$ ésimo EU e $\boldsymbol{z}_{u} \in \mathbb{C}^{N_{U} \times 1}$ é o ruído aditivo modelado como um vetor aleatório gaussiano complexo simétrico circular de média zero em cada u-ésimo EU (Cho et al., 2010).

A Figura 2 esboça o modelo de canal de enlace de descida para um sistema MIMO multiusuário.

A técnica de Diagonalização de Blocos é adequada em cenários onde os EUs possuem múltiplas antenas. Nessa técnica, todos os sinais não desejados são interpretados como interferência e são cancelados. A interferência causada pelos sinais de outros EUs e a interferência entre as antenas de um mesmo EU são canceladas por précodificação e técnicas de detecção de sinais, respectivamente (Cho et al., 2010). 


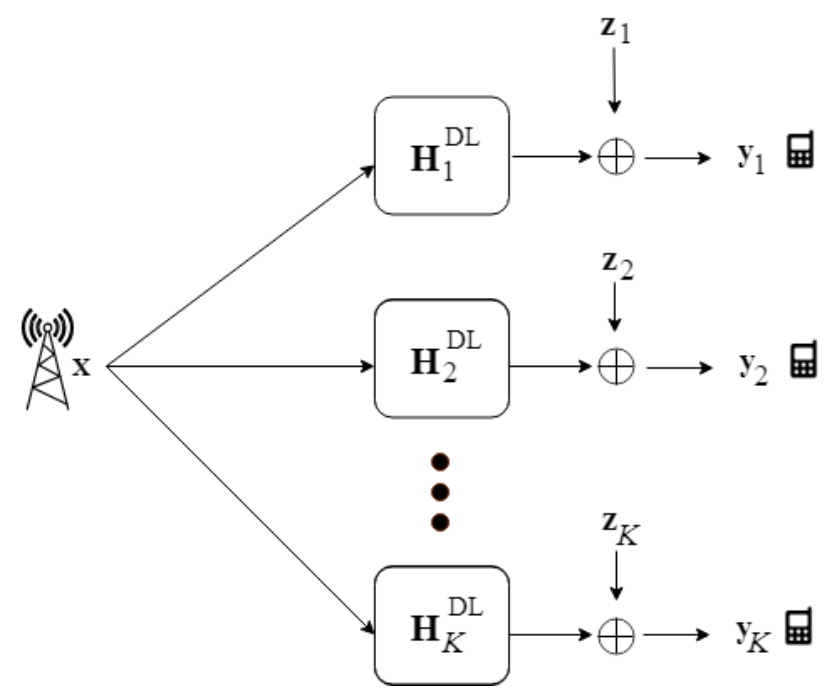

Figura 2. Modelo de canal downlink para sistema MIMO multiusuário: Canal Broadcast (BC). Adaptado de (Cho et al., 2010).

Seja $N_{M, u}$ o número de antenas para o u-ésimo EU, $u=1,2, \ldots, K$. Para o sinal do $u$-ésimo $\mathrm{EU} \tilde{\boldsymbol{x}}_{u} \in \mathbb{C}^{N_{U} \times 1}$, o sinal recebido $\boldsymbol{y}_{u} \in \mathbb{C}^{N_{U, u} \times 1}$ é dado pela Equação (3).

$$
\boldsymbol{y}_{u}=\boldsymbol{H}_{u}^{D L} \boldsymbol{W}_{u} \tilde{\boldsymbol{x}}_{u}+\sum_{k=1, k \neq u}^{K} \boldsymbol{H}_{u}^{D L} \boldsymbol{W}_{k} \tilde{\boldsymbol{x}}_{k}+\boldsymbol{z}_{u}
$$

onde $\boldsymbol{W}_{u} \in \mathbb{C}^{N_{B} \times N_{M, u}}$ é a matriz de pré-codificação para o u-ésimo EU.

\section{ALOCAÇÃO DE POTÊNCIA EM SISTEMAS MIMO MULTIUSUÁRIO}

Para o sistema NOMA MIMO abordado, são considerados dois métodos de alocação de potência: o primeiro método é fundamentado na proposta de (Ali et al., 2016a) e o segundo método é calcado no trabalho de (Zamani et al., 2018). São chamados neste de artigo de Algoritmo Adaptativo 1 e Adaptativo 2, respectivamente.

Para o primeiro método, consideremos que os ganhos de canal normalizados resultantes definidos pelos $K$ usuários sejam $g_{1}, g_{2}, \ldots, g_{K}$, onde $g_{1}>g_{2}>\ldots>g_{K}$. Os respectivos requisitos de vazão para todos os $K$ usuários são assumidos como $R_{1}, R_{2}, \ldots R_{K}$, onde $R_{1}>0, \forall k$ e $R_{k}$ é expresso pela Equação (4) ((Ali et al., 2016b)) ((Ali et al., 2016a)).

$$
R_{k}=B \log _{2}\left(1+\frac{g_{k} p_{k}}{g_{k} \sum_{j=1}^{k-1} p_{j}+1}\right)
$$

onde $B$ é a largura de banda efetiva dos sistema, $g_{k}$ é o ganho no $k$-ésimo usuário e $p_{k}$ é a potência do $k$-ésimo usuário.

Se o número de usuários NOMA MIMO for igual ao número de antenas de transmissão da $\mathrm{EB}$, então a largura de banda total do sistema $B$ será utilizada para servir todos os usuários do sistema. Logo, o problema de alocar a potência ótima para os usuários pode ser formulada da seguinte forma:

$$
\underset{p}{\operatorname{maximizar}} \sum_{k=1}^{K} B \log _{2}\left(1+\frac{g_{k} p_{k}}{g_{k} \sum_{j=1}^{k-1} p_{j}+1}\right)
$$

sujeito a:

$$
\begin{aligned}
& \mathrm{C} 1: \sum_{k=1}^{K} p_{k} \leq p \\
& \mathrm{C} 2: B \log _{2}\left(1+\frac{g_{k} p_{k}}{g_{k} \sum_{j=1}^{k-1} p_{j}+1}\right) \geq R_{k} \\
& \mathrm{C} 3:\left(p_{k}-\sum_{j=1}^{k-1} p_{j}\right) g_{k-1} \geq p_{t o l}, \forall k \neq 1
\end{aligned}
$$

onde $p_{t o l}$ é diferença mínima de potência entre diferentes usuários para o SIC ser realizado e $p$ é a potência total alocada para os usuários do sistema NOMA MIMO. O problema de otimização em (5) é análogo à alocação de potência para o enlace de descida NOMA para antena única na EB apresentado em Ali et al. (2016b). Em (Ali et al., 2016a), são propostas equações para solucionar o problema de otimização em (5) para um sistema NOMA MIMO. Segundo esses autores, a potência para o usuário com menor ganho de canal é dada pela Equação (6) (Ali et al., 2016a):

$$
\begin{gathered}
p_{1}=\frac{p}{\prod_{\substack{j=2 \\
j \notin B^{\prime}}}^{K} \varphi_{j} \prod_{\substack{j=2 \\
j \in B^{\prime}}}^{K} 2}-\sum_{\substack{j=2 \\
j \notin B^{\prime}}}^{K} \frac{\left(\varphi_{j}-1\right)}{\prod_{\substack{j^{\prime}=2 \\
j^{\prime} \notin B^{\prime}}}^{j} \varphi_{j^{\prime}} \prod_{\substack{j^{\prime}=2 \\
j^{\prime} \in B^{\prime}}}^{j} 2}- \\
\sum_{\substack{j=2 \\
j \notin C^{\prime}}}^{K} \frac{p_{t o l}}{2 g_{j-1} \prod_{\substack{j-1 \\
j^{\prime}=2 \\
j^{\prime} \notin B^{\prime}}}^{\substack{j-1 \\
j^{\prime}}} \prod_{\substack{j^{\prime}=2 \\
j^{\prime} \in B^{\prime}}}^{j-1} 2}
\end{gathered}
$$

Para os demais usuários do sistema NOMA MIMO, a alocação ótima de potência adaptativa pode ser expressa pela Equação (7) (Ali et al., 2016a):

$$
\begin{array}{r}
p_{k}=\left[\frac{p}{\prod_{\substack{j=k \\
j \notin B^{\prime}}}^{K} \varphi_{j} \prod_{\substack{j=k \\
j \in B^{\prime}}}^{K} 2}-\sum_{\substack{j=k \\
j \notin B^{\prime}}}^{K} \frac{\left(\varphi_{j}-1\right)}{\prod_{\substack{j^{\prime}=k \\
j^{\prime} \notin B^{\prime}}}^{j} \varphi_{j^{\prime}} \prod_{\substack{j^{\prime}=k \\
j^{\prime} \in B^{\prime}}}^{j} 2}-\right. \\
\left.\sum_{\substack{j=k \\
j \notin C^{\prime}}}^{K} \frac{p_{\text {tol }}}{2 g_{j-1} \prod_{\substack{j^{\prime}=k \\
j^{\prime} \notin B^{\prime}}}^{j-1} \varphi_{j^{\prime}} \prod_{\substack{j^{\prime}=k \\
j^{\prime} \in B^{\prime}}}^{j-1} 2}+\frac{\delta}{g_{k}}\right] \times \\
\left(\delta \varphi_{k}+(-1)^{\delta}\right)+\frac{p_{t o l}(1-\delta)}{g_{k-1}}
\end{array}
$$

onde $B^{\prime}$ e $C^{\prime}$ são os conjuntos complementares dos requisitos de taxa mínima dos usuários e das restrições SIC, respectivamente, os quais são definidos em (Ali et al., 2016b). A variável $\varphi_{k}=2^{\frac{R_{k}}{B}}$ também é definida em (Ali 
et al., 2016b). A variável $\delta$ faz o seguinte controle: se $\delta=1$, então $k \notin B^{\prime}$ e se $\delta=0$, então $k \in B^{\prime}$.

Já para o segundo método de alocação de potências, o problema de otimização para maximizar a vazão do sistema NOMA MIMO é descrito pela Equação (8) (Zamani et al., 2018):

$\underset{p}{\operatorname{maximizar}} \sum_{k=1}^{K} \log _{2}\left(1+\frac{p_{k}\left|\hat{g}_{k}\right|^{2}}{\left|\hat{g}_{k}\right|^{2} \sum_{j=1}^{k-1} p_{j}+p \sigma_{\epsilon}^{2}+\sigma^{2}}\right)$

sujeito a:

$\mathrm{C} 1: \sum_{k=1}^{K} p_{k} \leq p$

$\mathrm{C} 2: p_{k} \geq\left(\omega_{j}-1\right)\left(\sum_{j=1}^{k-1} p_{j}+\frac{p \sigma_{\epsilon}^{2}+\sigma^{2}}{\left|\hat{g}_{k}\right|^{2}}\right), \forall k=1,2, \ldots, K$.

onde $\omega_{j}=2^{R_{j}^{\text {min }}}, R_{j}^{\text {min }}$ é a taxa de dados mínima exigida para o j-ésimo usuário, $\hat{g}_{k}$ é o ganho estimado do $k$-ésimo usuário, $p$ é a potência total alocada para os usuários, $\sigma_{\epsilon}^{2}$ é a variância da distribuição Gaussiana complexa do erro de estimação do canal e $\sigma^{2}$ é a variância do ruído Gaussiano branco aditivo complexo.

De acordo com (Zamani et al., 2018), as condições de Karush-Kuhn-Tucker (KKT) são necessárias e suficientes para obter a solução ótima do problema de otimização em (8). Assim, as alocações de potência ótimas para o usuário com menor ganho $(k=1)$ de canal e para os demais usuários do sistema NOMA MIMO $(k=2,3, \ldots, K)$ são descritas pelas Equações (9) e (10), respectivamente:

$$
\begin{aligned}
p_{1}= & \frac{p}{\prod_{k=2}^{K} \omega_{k}}-\sum_{j=2}^{K} \frac{\left(\omega_{j}-1\right)\left(\sigma_{\epsilon}^{2} p+\sigma^{2}\right)}{\prod_{l=2}^{j} \omega_{l}\left|\hat{g}_{k}\right|^{2}} \\
p_{k}= & \frac{\left(\omega_{k}-1\right) p}{\prod_{j=k}^{K} \omega_{j}}+\frac{\left(\omega_{k}-1\right)\left(\sigma_{\epsilon}^{2} p+\sigma^{2}\right)}{\omega_{k}\left|\hat{g}_{k}\right|^{2}}- \\
& \sum_{j=k+1}^{K} \frac{\left(\omega_{k}-1\right)\left(\omega_{j}-1\right)\left(\sigma_{\epsilon}^{2} p+\sigma^{2}\right)}{\prod_{l=k}^{j} \omega_{l}\left|\hat{g}_{k}\right|^{2}}
\end{aligned}
$$

5.1 Algoritmo de Alocação de Potência com Seleção de Usuários

Nesta seção, propomos um algoritmo de alocação adaptativa de potência que considera além das condições do canal, a seleção de usuários para prover aumento de taxa de transmissão total do sistema. Assim, de acordo com as condições do canal para o usuário, sua potência será dinamicamente ajustada. Segundo (Cho et al., 2010), a seguinte condição deve ser respeitada na Diagonalização de Blocos para que o sistema não sofra com interferências severas:

$$
N_{K} \leq \frac{N_{T}}{N_{R}}
$$

onde $N_{K}$ é o total de usuários selecionados, $N_{T}$ é o total de antenas de transmissão e $N_{R}$ é o número de antenas de recepção em cada usuário.

Nesse sentido, propõe-se realizar alocação dinâmica de potência obedecendo a condição apresentada na Equação (11), utilizando seleção de usuários. A seleção de usuários poderia ser feita por busca exaustiva. Entretanto, propõe-se neste artigo a utilização de um método iterativo, onde seleciona-se os usuários com maior projeção sobre o espaço ortogonal aos usuários previamente selecionados. Esse método iterativo se baseia no trabalho de projeções sucessivas de (Yoo and Goldsmith, 2006), mas onde não foi considerada a técnica NOMA. Inicialmente, considerase todos os usuários como candidatos e, em seguida, seleciona-se o usuário com maior ganho e atualiza-se o conjunto de usuários selecionados. Na primeira iteração, calcula-se o espaço ortogonal aos usuários selecionados e realiza-se a projeção do vetor de ganho de cada candidato nesse espaço. Por fim, seleciona-se o candidato com maior projeção e atualiza-se o conjunto de usuários selecionados. O procedimento é repetido até que se tenha completado as seleções (Yoo and Goldsmith, 2006).

Essa sequência de passos pode ser matematicamente descrita pelas Equações (12) à (14) (Yoo and Goldsmith, 2006):

$$
\boldsymbol{H}=\left[\boldsymbol{h}_{\xi_{1}^{T}}, \ldots, \boldsymbol{h}_{\xi_{|\xi|}^{T}}^{T}\right]
$$

$$
\begin{gathered}
\boldsymbol{N}=\boldsymbol{I}-\boldsymbol{H}\left(\boldsymbol{H}^{H} \boldsymbol{H}\right)^{-1} \boldsymbol{H}^{H} \\
\zeta_{i}=\left\|\boldsymbol{N} \boldsymbol{h}_{i}^{T}\right\|^{2}, \forall i \in \Omega \xi
\end{gathered}
$$

onde $\boldsymbol{H}$ é a matriz de ganhos dos usuários selecionados que, a cada iteração, aumenta sua dimensão em 1 até que $|\xi|=N, \boldsymbol{h}_{\xi_{i}}=\left[h_{1, \xi i}, \ldots, h_{N_{T}, \xi i}\right], \boldsymbol{N}$ é o espaço ortogonal aos usuários selecionados, calculado por meio da subtração da matriz identidade pela matriz pseudo-inversa do canal aplicada à matriz do canal. $\xi_{i}$ corresponde à métrica de seleção e é calculada pela norma da projeção do vetor do $i$-ésimo usuário sobre o espaço $N$, onde $\xi=\xi \cup\{k\}$. A variável $\Omega$ representa o conjunto de índices de usuários candidatos. Por fim, $k$ é o índice do usuário selecionado na iteração, dado por $k=i \zeta_{i}$.

O procedimento proposto realizado para a alocação adaptativa de potência seguiu os passos descritos no Algoritmo 1. No Algoritmo 1, a cada iteração atualizase as potências dos usuários de acordo com o Algoritmo Adaptativo 2. Caso a condição descrita pela Equação (11) não seja respeitada, seleciona-se os melhores usuários de acordo com as Equações (12) à (14). 
Entrada: total_pacotes, $S N R, p, N_{A}, N_{K}, N_{T}, N_{R}$ 1 para cada $s \in S N R$ faça

$2 \quad$ para cada $t \in$ total_pacotes faça

$3 \quad \mid \quad$ Seja $g_{k}(t)$ uma variável aleatória representando o ganho de canal;

se $N_{K} \times N_{R} \leq N_{T}$ então

4

Dados $S N R(s), g_{k}(t), N_{R}, N_{T}$, calcule $p_{k=1}(t)$ com a Equação (9)

Dados $S N R(s), g_{k}(t), N_{R}, N_{T}$, calcule $p_{k>1}(t)$ com a Equação (10)

$6 \quad$ fim

Dados $N_{K}, N_{A}, g_{k}(t)$ selecione os usuários com maiores valores de $\xi$, calculados com as Equações (12) à (14)

Dados $S N R(s), g_{k}(t), N_{R}, N_{T}$, calcule $p_{k=1}(t)$ com a Equação $(9)$

Dados $S N R(s), g_{k}(t), N_{R}, N_{T}$, calcule $p_{k>1}(t)$ com a Equação (10)

Algorithm 1. AlocaÇão de PotênCia e Seleção de Usuários.

onde $N_{A}$ é o número de usuários ativos, $N_{K}$ é o número de usuários do sistema, $N_{R}$ é o número de antenas de recepção para cada usuário, $N_{T}$ é o número de antenas de transmissão na EB, $p$ é a potência total alocada para o sistema, $p_{k}$ é a potência alocada para o $k$-ésimo usuário, $g_{k}$ é o ganho de canal do k-ésimo usuário, total_pacotes é o total de pacotes a serem transmitidos no sistema e $S N R$ é o valor da relação sinal-ruído considerada em cada iteração do algoritmo. Assim, o algoritmo selecionará todos os usuários quando $N_{K} \times N_{R} \leq N_{T}$ e, caso contrário, selecionará os $N_{A}$ melhores usuários de acordo com o algoritmo de Projeções Sucessivas.

\section{SIMULAÇÕES E RESULTADOS}

Para avaliar o desempenho da proposta de alocação adaptativa de potência com seleção de usuários, desenvolveuse um programa em MATLAB ${ }^{\circledR}$ capaz de simular quatro cenários para a alocação de potência dos usuários: fixa, aleatória e adaptativa 1 e adaptativa 2 . Incialmente, considerou-se um sistema consistindo de uma EB com 20 antenas transmissoras enviando dados em um enlace de descida para 20 EUs com duas antenas receptoras cada. A Tabela 1 apresenta os parâmetros utilizados nas simulações.

Para a simulação com alocação aleatória de potência, gerou-se valores aleatórios de potência para cada usuário em cada iteração sobre o tempo total. Para a simulação com alocação fixa de potência, alocou-se valores fixos iguais a $p /\left(N_{K} N_{R}\right)$ para todos os usuários do sistema. A execução do algoritmo foi feita levando-se em consideração diferentes cenários.

Os resultados de taxa agregada (sum rate) para simulação sem a seleção de usuários e utilizando o algoritmo proposto são apresentados na Figura 3. A taxa agregada corresponde ao total de bits transmitidos pelos usuários no sistema.

Pelos resultados apresentados, é possível notar na simulação sem a seleção de usuários que a taxa agregada sofre reduções severas quando a condição (11) não é respeitada, para a configuração do sistema considerada na Figura 3. Assim, a aplicação do algoritmo proposto de alocação adaptativa com seleção de usuários permite que mais usuários consigam fazer parte do sistema com valores de sum rate iguais ou maiores aos obtidos para o caso com a seleção de todos os usuários. Nota-se também que o algoritmo de alocação de potência Adaptativo 2 foi o que obteve maiores valores de sum rate quando não se considera seleção de usuários, principalmente com o aumento da quantidade de usuários.

Tabela 1. Parâmetros utilizados nas simulações.

\begin{tabular}{|l|c|}
\hline \multicolumn{1}{|c|}{ Parâmetro } & Valor \\
\hline Modulação & QPSK \\
\hline Número de frames $\left(N_{F}\right)$ & 10 \\
\hline Número de pacotes $\left(N_{P K}\right)$ & 200 \\
\hline Número de EU $\left(N_{K}\right)$ & 20 \\
\hline Número de antenas de recepção em cada EU $\left(N_{R}\right)$ & 2 \\
\hline Número de EB $\left(N_{E B}\right)$ & 1 \\
\hline Número de antenas de transmissão na EB $\left(N_{T}\right)$ & 20 \\
\hline Largura de banda de um bloco de recurso $(B)$ & $180 \mathrm{kHz}$ \\
\hline Potência Total do sistema $(p)$ & $5 \mathrm{~dB}$ \\
\hline SNR & 0 até $40 \mathrm{~dB}$ \\
\hline
\end{tabular}

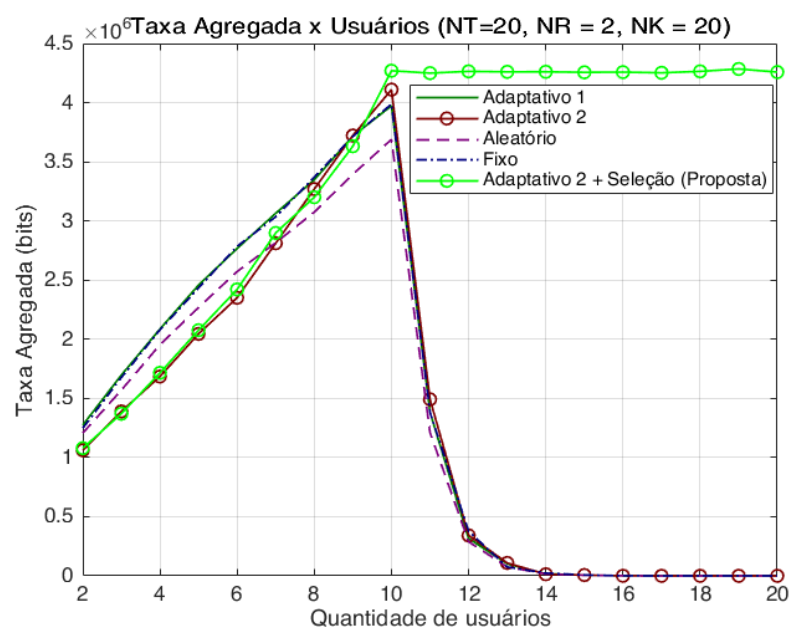

Figura 3. Taxa agregada para o cenário com $N_{T}=20$, $N_{K}=20, N_{R}=2$, com seleção de todos os usuários.

O gráfico da relação BER (do inglês, Bit Error Rate) versus SNR para os algoritmos de alocação de potência com e sem a seleção de usuários é apresentado na Figura 4. A partir dessa figura, é possível constatar que o método de transmissão por Diagonalização de Blocos com ZeroForcing de fato é capaz de cancelar a interferência entre os usuários, uma vez que a BER é reduzida com o aumento da SNR. Porém, a BER fica elevada quando a condição (11) não é satisfeita. Assim a seleção de usuários se torna eficiente quando combinada com os algoritmos de alocação de potência. 
O gráfico da vazão total do sistema versus SNR é apresentado na Figura 5. Como apresentado na Figura 4, a BER aumenta consideravelmente quando a condição (11) não é satisfeita. Consequentemente, a vazão total dos usuários para esse caso fica praticamente nula, conforme pode ser visualizado na Figura 5. Assim, utilizando-se o algoritmo proposto, a vazão total do sistema aumenta significativamente.

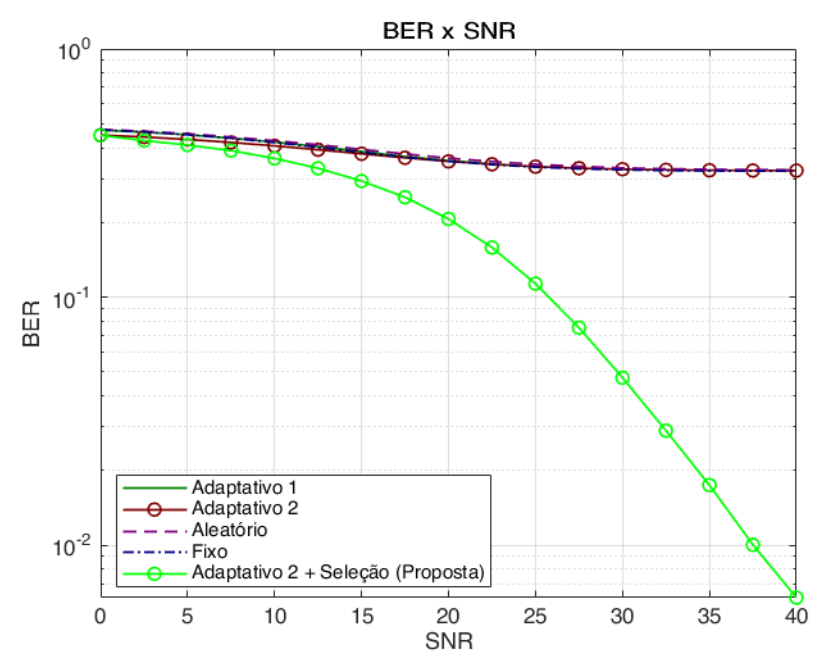

Figura 4. Relação BER versus SNR para o cenário com $N_{T}=20, N_{K}=20, N_{R}=2$, com a seleção dos 10 melhores usuários no Algoritmo Proposto.

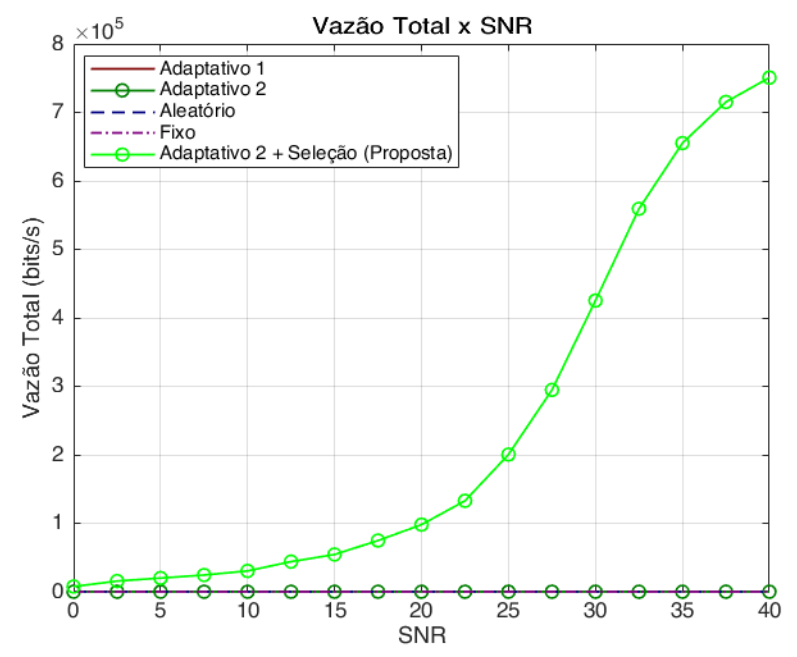

Figura 5. Relação Vazão total versus SNR para o cenário com $N_{T}=20, N_{K}=20, N_{R}=2$, com a seleção dos 10 melhores usuários no Algoritmo Proposto.

Com o objetivo de avaliar o comportamento da taxa agregada com o aumento dos usuários e número de antenas, dado o crescente interesse em pesquisas por MIMO Massivo (Mundy, 2019), realizou-se uma simulação com a variação dos usuários de 2 até 64 , selecionando os 32 melhores. O resultado dessa simulação é apresentado na Figura 6 . Como a proposta de utilização do algoritmo Adaptativo 2 com seleção de usuários obtém os menores valores de BER (vide Figura 4), consequentemente o algoritmo proposto obtém maiores valores de sum rate, conforme indicado pela Figura 6.

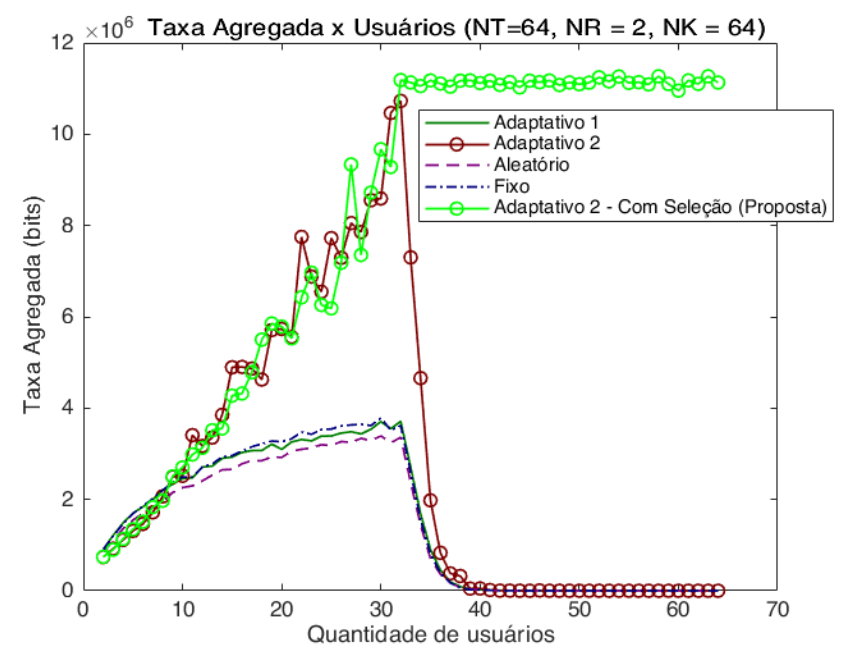

Figura 6. Taxa agregada para o cenário com $N_{T}=64$, $N_{K}=32,2 \leq N_{R} \leq 64$, com seleção dos 32 melhores usuários no Algoritmo Proposto.

\section{CONCLUSÃO}

A combinação das técnicas NOMA com sistemas MIMO multiusuário em sistemas de comunicação móvel tem sido considerada uma abordagem promissora para as redes $5 \mathrm{G}$ em vários estudos realizados. A eficiência na seleção de usuários e na alocação de potências para os mesmos em uma célula são fatores fundamentais para a melhoria do desempenho de sistemas NOMA MIMO.

Neste trabalho, é proposto um algoritmo para seleção de usuários e alocação adaptativa de potência considerando o enlace de descida de um sistema NOMA MIMO. Para analisar o comportamento da variação do número de usuários no sistema, foram considerados nas simulações cenários com diferentes quantidades de usuários no sistema. A escolha dos usuários foi realizada de maneira a maximizar a capacidade do sistema de acordo com Yoo and Goldsmith (2006). Os algoritmos propostos para a alocação adaptativa de potência também foram desenvolvidos com base em problemas de otimização, cujos objetivos são o de maximizar a vazão dos usuários Ali et al. (2016a) Zamani et al. (2018).

Para fins de comparação, implementou-se um simulador de sistema NOMA MIMO em MATLAB ${ }^{\circledR}$ capaz de simular quatro métodos de alocação de potência: aleatória, fixa e adaptativa 1 e adaptaiva 2. Os resultados das simulações realizadas indicaram que o método de alocação de potência adaptativa 2 foi o que obteve maiores valores de vazão, quando não se considera seleção de usuários. Quando comparado a outros algoritmos de alocação de potência, o algoritmo proposto (contemplando seleção de usuários) apresenta resultados superiores aos demais métodos de alocação de potência em termos de taxa agregada, BER e vazão. Constatou-se também que o aumento de usuários sem respeitar os limites da Diagonalização de Blocos provoca aumento da BER e, consequentemente, reduz drasticamente a vazão. Quando o algoritmo de 
seleção de usuários via projeções sucessivas é utilizado, esse problema é eliminado, permitindo que vários usuários consigam transmitir dados mantendo a vazão em valores satisfatórios.

O modelo NOMA MIMO considerado neste trabalho se refere a um cenário com uma única célula. Sistemas com múltiplas células podem adicionar mais interferência, podendo piorar o desempenho da vazão do sistema. Portanto, como trabalhos futuros, pode-se avaliar a abordagem de alocação adaptativa de potência proposta neste artigo em cenários com múltiplas células.

\section{REFERÊNCIAS}

Ali, S., Hossain, E., and Kim, D.I. (2016a). Nonorthogonal multiple access (noma) for downlink multiuser mimo systems: User clustering, beamforming and power allocation. IEEE Access.

Ali, S., Tabassum, H., and Hossain, E. (2016b). Dynamic user clustering and power allocation for uplink and downlink non-orthogonal multiple access (noma) systems. IEEE Access.

Cho, Y.S., Kim, J., Yang, W.Y., and Kang, C.G. (2010). MIMO-OFDM Wireless Communication with MATLAB, volume 1. John Willey \& Sons, 1st edition.

Ding, Z., Adachi, F., and Poor, H.V. (2016). The application of mimo to non-orthogonal multiple acceess.

Islam, S.M.R., Zeng, M., and Dobre, O.A. (2017). Noma in $5 \mathrm{~g}$ systems: Exciting possibilities for enhancing spectral efficiency.

Kizilirmak, R.C. (2016). Towards 5g wireless networks - a physical layer perspective. InTechOpen, 1(1), 83-98.

Luo, F.L. and Zhang, C. (2016). Signal Processing For 5G: Algorithms and Implementations. John Willey \& Sons, 1st edition.

Mundy, J. (2019). What is massive mimo technology? https://5g.co.uk/guides/ what-is-massive-mimo-technology/. Acessado: 2019-05-05.

Saito, Y., Kishiyama, Y., Benjebbour, A., Nakamura, T., Li, A., and Higuchi, K. (2013). Non-orthogonal multiple access (noma) for cellular future radio access. IEEE $V T C$.

Yoo, T. and Goldsmith, A. (2006). On the optimality of multiantenna broadcast scheduling using zero-forcing beamforming. IEEE Journal on Selected Areas in Communications, 528-541.

Zamani, M.R., Eslami, M., and Khorramizadeh, M. (2018). Optimal sum-rate maximization in a noma system with channel estimation error. 26th ICEE (Iranian Conference on Electrical Engineering), 720-724.

Zeng, M., Yadav, A., Dobre, O.A., Tsiropoulos, G.I., and Poor, H.V. (2017). On the sum rate of mimo-noma and mimo-oma systems. 